\title{
Avaliação da adesão ao checklist de cirurgia segura em um hospital universitário
}

\begin{abstract}
Assessment of adherence to safe surgery checklist in a university hospital
\end{abstract}
Evaluación de la adherencia a la lista de verificación de cirugía segura en un hospital universitario

\author{
Tânia Solange Bosi de Souza MagnagoI, Taís Carpes Lanes ${ }^{\text {II }}$, Naiane Glaciele da Costa \\ Gonçalves $^{\mathrm{III}}$, Juliana Dal Ongaro ${ }^{\mathrm{IV}}$, Franciele Ormizinda Almeidav ${ }^{\mathrm{v}}$, \\ Graziele de Lima Dalmolin ${ }^{\mathrm{VI}}$
}

Resumo: Objetivo: avaliar a adesão ao checklist de cirurgia segura em um hospital universitário do Sul do Brasil. Método: estudo transversal descritivo, retrospectivo, realizado nas unidades de internação e centro cirúrgico de um hospital universitário do Rio Grande do Sul, Brasil. A coleta foi feita nos prontuários de pacientes submetidos a cirurgias eletivas entre os meses de setembro e dezembro de 2016. A mensuração da taxa de adesão foi mediante a existência e qualidade do preenchimento dos itens do checklist, empregando-se estatística descritiva. Resultados: a taxa de adesão ao checklist nas unidades de internação variou de 23,3 a 74,4\% e no centro cirúrgico, de 55,2 a 61,2\%. Conclusão: evidenciou-se baixa adesão ao preenchimento do checklist, sendo importante a implementação de estratégias que possam auxiliar em seu preenchimento.

Descritores: Segurança do Paciente; Lista de Checagem; Centros Cirúrgicos; Enfermagem

Abstract: Objective: To assess adherence of the safe surgery checklist in a university hospital in the South of Brazil. Method: a descriptive retrospective cross-sectional study conducted in the care units and operating room of a university hospital in Rio Grande do Sul, Brazil. Data were collected from medical records of patients undergoing elective surgery between September and December 2016. The adherence rate was measured by the existence and

\footnotetext{
I Enfermeira. Professora. Doutora em Enfermagem. Universidade Federal de Santa Maria. Santa Maria, RS, Brasil. E-mail: tmagnago@terra.com.br. Orcid: https://orcid.org/0000-0002-5308-1604.

II Enfermeira. Mestranda em Enfermagem. Universidade Federal de Santa Maria. Santa Maria, RS, Brasil. E-mail: taislanes_rock@hotmail.com. Orcid: https://orcid.org/0000-0001-9337-7875.

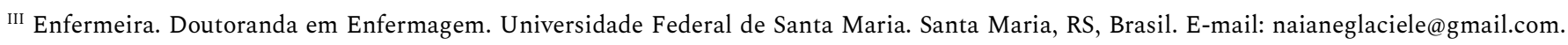
Orcid: https://orcid.org/0000-0003-2582-2417.

IV Enfermeira. Mestranda em Enfermagem. Universidade Federal de Santa Maria. Santa Maria, RS, Brasil. E-mail: dongaro.ju@gmail.com. Orcid: https://orcid.org/0000-0003-0058-0132.

v Acadêmica de Enfermagem. Universidade Federal de Santa Maria. Santa Maria, RS, Brasil. E-mail: franciele11041994@gmail.com. Orcid: https://orcid.org/0000-0001-6771-3863.

VI Enfermeira. Professora. Doutora em Enfermagem. Universidade Federal de Santa Maria. Santa Maria, RS, Brasil. E-mail: grazi.dalmolin@gmail.com. Orcid: https://orcid.org/0000-0003-0985-5788.
} 
Avaliação da adesão ao checklist de cirurgia segura em um hospital universitário | 2

quality of filling the checklist items, using descriptive statistics. Results: the rate of adherence to the checklist in the care units ranged from 23.3 to $74.4 \%$ and in the operating room, from 55.2 to $61.2 \%$. Conclusion: there was low adherence to filling of the checklist, being important the implementation of strategies that can assist in its filling.

Descriptors: Patient Safety; Checklist; Surgicenters, Nursing

Resumen: Objetivo: evaluar la adhesión al checklist de cirugía segura en un hospital universitario en el sur de Brasil. Método: estudio transversal descriptivo, retrospectivo, realizado en las unidades de internación y centro quirúrgico de un hospital universitario en Rio Grande do Sul, Brasil. La colecta fue realizada en los prontuarios de pacientes sometidos a cirugías electivas entre los meses de septiembre y diciembre de 2016. La verificación de la tasa de adhesión fue por medio de la existencia y la calidad de la cumplimentación de los ítems del checklist, utilizando estadística descriptiva. Resultados: la tasa de adhesión al checklist en las unidades de internación osciló entre 23,3 y 74,4\% y en el centro quirúrgico, de 55,2 a 61,2\%. Conclusión: se evidenció baja adhesión a la cumplimentación del checklist, siendo importante la implementación de estrategias que puedan ayudar en su cumplimentación.

Descriptores: Seguridad del Paciente; Lista de Verificación; Centros Quirúrgicos; Enfermería

\section{Introdução}

As implicações associadas aos procedimentos cirúrgicos são constantes e representam, mundialmente, um problema de saúde..$^{1-2} \mathrm{Um}$ a cada trezentos pacientes admitidos nos hospitais morre e, em mais de 50\%, a causa da morte está relacionada a eventos adversos cirúrgicos evitáveis. ${ }^{3}$ No Brasil, ainda há poucos dados, mas os existentes são alarmantes. Estudo realizado em um hospital universitário do Sul do Brasil evidenciou a ocorrência de 98,7\% ( $\mathrm{n}=2.396)$ de incidentes sem danos e 1,3\% $(\mathrm{n}=27)$ com danos aos pacientes em uma unidade clínica círurgica. ${ }^{4}$

Na perspectiva de sensibilizar os profissionais da saúde para uma assistência segura foi desenvolvido, a partir da Aliança Mundial de Saúde, o segundo desafio global entre 2007 e 2008, intitulado "Cirurgias seguras salvam vidas". ${ }^{1}$ Nesse desafio, foi proposto o Manual para Cirurgia Segura, objetivando a redução da mortalidade por eventos adversos evitáveis nos pacientes cirúrgicos. Nele, consta uma lista de verificação (checklist) formada por um conjunto de ações que devem ser realizadas, checadas e registradas durante o processo cirúrgico. ${ }^{1}$

O checklist auxilia a equipe na condução das ações cirúrgicas e contribui na diminuição de riscos de danos aos pacientes, oferecendo aos profissionais uma prática mais segura..$^{5-6}$ Para isso, é necessário que a equipe efetue o preenchimento por completo dos itens dispostos nessa 
3 | Magnago TSBS, Lanes TC, Gonçalves NGC, Ongaro JD, Almeida FO, Dalmolin GL

ferramenta, alcançando a adesão conforme o preconizado. Estes itens estão divididos em fases: antes da indução anestésica, antes da incisão cirúrgica e antes de retirar o paciente da sala de operação. ${ }^{1}$

O hospital cenário deste estudo fez a implantação do checklist de cirurgia segura em fevereiro de 2016, com formulário de preenchimento manual. Percebeu-se ser necessário realizar um diagnóstico institucional, para identificar a adesão dos profissionais ao checklist e, posteriormente, sinalizar as facilidades e fragilidades em seu preenchimento. ${ }^{7}$ Frente ao exposto, tem-se como questão de pesquisa: "Qual é a taxa de adesão ao checklist de cirurgia segura de um hospital universitário do Sul do Brasil?” Para responder a esse questionamento, objetivou-se avaliar a adesão ao checklist de cirurgia segura em um hospital universitário do Sul do Brasil.

\section{Método}

Estudo transversal descritivo, retrospectivo, desenvolvido no centro cirúrgico e unidades de internação de um hospital universitário de grande porte, localizado no Estado do Rio Grande do Sul, Brasil. O hospital possui 403 leitos para internação, com 100\% dos atendimentos realizados pelo Sistema Único de Saúde (SUS). O centro cirúrgico é composto por sete salas, recebe pacientes de 18 unidades de internação e do pronto-socorro da instituição. No ano de 2016, foram realizadas 7.376 cirurgias (média de 615 cirurgias/mês).

Considerou-se como população todos os prontuários de pacientes submetidos à cirurgia eletiva, no período de setembro a dezembro de 2016. Para fins estatísticos, foi calculada uma amostra mínima por meio de uma fórmula para amostragem finita. Considerando-se o total de 1767 cirurgias eletivas realizadas no período de setembro a dezembro de 2016, um erro amostral de $5 \%$ e uma proporção estimada de $50 \%$ de checklists preenchidos, estabeleceu-se um mínimo de 317 checklists para análise. ${ }^{8}$ 
Avaliação da adesão ao checklist de cirurgia segura em um hospital universitário | 4

A partir da lista de marcação de cirurgia, adotou-se como critério de inclusão: prontuários dos pacientes submetidos a cirurgias eletivas no período de setembro a dezembro de 2016. Foram excluídos os prontuários não localizados no setor de arquivo, ou seja, por estarem no setor de faturamento, por exemplo.

O checklist adotado na instituiçãa pesquisada é uma adaptação do modelo preconizado pela Organização Mundial de Saúde (OMS). Além dos itens preconizados pela OMS para "antes da indução", “antes da incisão” e "antes da saída da sala de cirurgia”, foram adicionados itens para o pré-operatório e para a chegada do paciente no centro cirúrgico. Ele é um formulário impresso, que após preenchido pelo técnico de enfermagem ou circulante de sala é anexado no prontuário do paciente.

A coleta de dados foi realizada durante o mês de dezembro de 2016, em turno matutino, de segunda a sexta-feira, por dois coletadores previamente capacitados. O instrumento de coleta dos dados foi elaborado com base no checklist da instituição, composto por quatro etapas: $1^{\mathbf{a}}$ ) Checklist pré-cirúrgico (realizado na unidade de internação); $2^{a}$ ) Recepção do paciente no centro cirúrgico; $\left.3^{\mathbf{a}}\right)$ Antes da indução anestésica e antes de iniciar a cirurgia; e $4^{\mathbf{a}}$ ) Antes de o paciente sair da sala (final da cirurgia).

Os dados foram digitados no Software Microsoft Excel ${ }^{\circledR}$, com verificação dos erros e inconsistências na digitação. Após, foi realizada a análise dos dados no Programa PASW 18.0 for Windows. As variáveis categóricas foram analisadas por meio da frequência absoluta $(\mathrm{N})$ e relativa $(\%)$.

O Cálculo de Adesão foi realizado pela razão entre o número de checklists preenchidos integralmente e o número total de checklists em cada mês. Expresso pela seguinte fórmula:

Adesão $(\%)=\underline{\text { número de checklists } \text { preenchidos integralmente no mês* }} 100$

Número de cirurgias eletivas no mês 
5 | Magnago TSBS, Lanes TC, Gonçalves NGC, Ongaro JD, Almeida FO, Dalmolin GL

A pesquisa recebeu apreciação pelo Comitê de Ética em Pesquisa (CEP): 1.812.525; e recebeu parecer favorável conforme o Certificado de Apresentação para Apreciação Ética (CAAE) nํ. 61553616.6.0000.5346, em 21 de outubro de 2016. Foram respeitados os aspectos éticos conforme a Resolução $n^{\circ}$ 466/ 2012 do Conselho Nacional de Saúde (CNS), garantindo o anonimato, a confidencialidade e a privacidade dos participantes. ${ }^{9}$

\section{Resultados}

No período de setembro a dezembro, foram realizadas 2310 cirurgias, destas, 574 no mês de setembro, 556 em outubro, 588 em novembro e 592 cirurgias em dezembro de 2016. Os checklists foram aplicados somente em cirurgias eletivas; e 76,4\% $(\mathrm{n}=1767)$ foram realizadas entre setembro a dezembro, compondo a população deste estudo, conforme a Tabela 1.

Tabela 1 - Distribuição dos checklists aplicados em cirurgias eletivas e taxa de adesão segundo mês de avaliação. 2016

\begin{tabular}{|c|c|c|c|c|c|c|c|}
\hline \multirow{3}{*}{ Mês } & \multirow{3}{*}{$\begin{array}{c}\text { Total de } \\
\text { cirurgias } \\
\text { realizadas } \\
\mathbf{N}\end{array}$} & \multirow{2}{*}{\multicolumn{2}{|c|}{$\begin{array}{c}\text { Total de } \\
\text { checklists de } \\
\text { cirurgias } \\
\text { eletivas }\end{array}$}} & \multicolumn{4}{|c|}{ Taxa de adesão ao checklist } \\
\hline & & & & \multicolumn{2}{|c|}{$\begin{array}{l}\text { Unidade de } \\
\text { internação }\end{array}$} & \multicolumn{2}{|c|}{ Centro cirúrgico } \\
\hline & & $\mathbf{N}$ & $\%$ & $\mathbf{N}^{* *}$ & $\%^{* *}$ & $\mathbf{N}^{* *}$ & $\%^{* *}$ \\
\hline Setembro & 574 & 409 & 71,3 & 100 & 24,4 & 234 & 55,2 \\
\hline Outubro & 556 & 425 & 76,6 & 316 & 74,4 & 260 & 61,2 \\
\hline Novembro & 588 & 470 & 80,1 & 318 & 67,7 & 271 & 57,7 \\
\hline Dezembro & 592 & 463 & 78,2 & 108 & 23,3 & 281 & 60,7 \\
\hline Total & 2310 & 1767 & 76,4 & 842 & 47,7 & 1046 & 59,3 \\
\hline
\end{tabular}

${ }^{*}$ Checklists totalmente preenchidos na unidade correspondente; ${ }^{* *}$ Corresponde ao número de checklists analisados no mês.

Fonte: Autores.

A taxa de adesão ao checklist nas unidades de internação variou de 23,3 a $74,4 \%$ e no centro cirúrgico, de 55,2 a 61,2\%. A avaliação da adesão ao preenchimento dos itens do checklist, 
Avaliação da adesão ao checklist de cirurgia segura em um hospital universitário | 6

na unidade de internação, a média quadrienal e o desvio padrão (DP) estão apresentados na Tabela 2.

Tabela 2 - Adesão ao checklist, por itens, nas unidades de internação durante o pré-operatório. 2016

\begin{tabular}{|c|c|c|c|c|c|c|c|c|c|c|c|}
\hline \multirow[t]{2}{*}{ Variáveis } & \multicolumn{2}{|c|}{ Set } & \multicolumn{2}{|c|}{ Out } & \multicolumn{2}{|c|}{ Nov } & \multicolumn{2}{|c|}{ Dez } & \multirow{2}{*}{$\frac{\text { Média }}{-}$} & \multirow{2}{*}{$\begin{array}{c}\text { DP } \\
-\end{array}$} & \multirow{2}{*}{$\begin{array}{c}\text { AQ } \\
\%\end{array}$} \\
\hline & $\mathbf{N}$ & $\%$ & $\mathbf{N}$ & $\%$ & $\mathbf{N}$ & $\%$ & $\mathbf{N}$ & $\%$ & & & \\
\hline Pulseira de Identificação & 319 & 78 & 333 & 78,4 & 368 & 78,3 & 363 & 78,4 & 346 & 20,4 & 78,3 \\
\hline Prontuário conferido & 238 & 58,2 & 239 & 56,2 & 270 & 57,4 & 258 & 55,7 & 251 & 13,4 & 56,9 \\
\hline $\begin{array}{l}\text { Exames de Imagens } \\
\text { impressos }\end{array}$ & 202 & 49,4 & 246 & 57,9 & 287 & 61,1 & 271 & 58,5 & 252 & 32,1 & 56,7 \\
\hline Paciente em jejum & 333 & 81,4 & 339 & 79,8 & 377 & 80,2 & 378 & 81,6 & 357 & 20,9 & 80,8 \\
\hline Utiliza anticoagulante & 289 & 70,7 & 320 & 75,3 & 350 & 74,5 & 355 & 76,7 & 329 & 26,4 & 74,3 \\
\hline Sítio demarcado & 250 & 61,1 & 256 & 60,2 & 308 & 65,5 & 310 & 67 & 281 & 28,1 & 63,5 \\
\hline Adornos retirados & 209 & 51,1 & 249 & 58,6 & 260 & 55,3 & 246 & 53,1 & 241 & 19,2 & 54,5 \\
\hline Utiliza prótese dentária & 327 & 80 & 329 & 77,4 & 360 & 76,6 & 369 & 79,7 & 346 & 18,5 & 78,4 \\
\hline Retirada de prótese dentária & 141 & 34,5 & 120 & 28,2 & 124 & 26,4 & 141 & 30,5 & 132 & 9,6 & 29,9 \\
\hline Higiene corporal & 301 & 73,6 & 309 & 72,7 & 347 & 73,8 & 340 & 73,4 & 324 & 19,6 & 73,4 \\
\hline Uso apenas da camisola & 216 & 52,8 & 249 & 58,6 & 255 & 54,3 & 227 & 49 & 237 & 15,9 & 53,7 \\
\hline Alérgico a medicações & 301 & 73,6 & 315 & 74,1 & 427 & 90,9 & 354 & 76,5 & 349 & 48,9 & 78,8 \\
\hline
\end{tabular}

AQ: Adesão quadrienal; Set: setembro; Out: outubro; Nov: novembro; Dez: dezembro.

Fonte: Autores.

O preenchimento do checklist era realizado nas unidades de internação durante o préoperatório antes do paciente ser encaminhado para o centro cirúrgico. De acordo com a Tabela 2, o item "paciente em jejum" obteve a maior média de adesão $(357 ; \mathrm{DP}=20,9)$ com taxa de $80,8 \%$ de preenchimento, e o de menor adesão foi "retirada de prótese dentária” $(132 ; \mathrm{DP}=9,6)$ com taxa de $29,9 \%$.

A avaliação da adesão ao preenchimento dos itens do checklist na porta de entrada do centro cirúrgico e na sala de cirurgia, bem como a média quadrienal e DP estão apresentados na Tabela 3.

Tabela 3 - Adesão ao checklist, por itens, na porta do centro cirúrgico e sala de cirurgia. 2016 
7 | Magnago TSBS, Lanes TC, Gonçalves NGC, Ongaro JD, Almeida FO, Dalmolin GL

\begin{tabular}{|c|c|c|c|c|c|c|c|c|c|c|c|}
\hline \multirow{3}{*}{$\begin{array}{l}\text { Variáveis } \\
\begin{array}{l}\text { Porta de entrada do Centro } \\
\text { cirúrgico }\end{array}\end{array}$} & \multicolumn{2}{|c|}{ Set } & \multicolumn{2}{|c|}{ Out } & \multicolumn{2}{|c|}{ Nov } & \multicolumn{2}{|c|}{ Dez } & \multirow{2}{*}{$\frac{\text { Média }}{-}$} & \multirow[t]{2}{*}{ DP } & \multirow{2}{*}{$\begin{array}{c}\mathrm{AQ} \\
\%\end{array}$} \\
\hline & $\mathbf{N}$ & $\%$ & $\mathbf{N}$ & $\%$ & $\mathbf{N}$ & $\%$ & $\mathbf{N}$ & $\%$ & & & \\
\hline & & & & & & & & & & & \\
\hline $\begin{array}{l}\text { Procedimento e sítio cirúrgico } \\
\text { confirmados com paciente e } \\
\text { equipe }\end{array}$ & 388 & 94,9 & 380 & 89,4 & 424 & 90,4 & 461 & 99 & 413 & 32,2 & 93,4 \\
\hline \multicolumn{12}{|l|}{ Sala de cirurgia } \\
\hline Equipe em sala sem adornos & 308 & 75,3 & 378 & 88,9 & 412 & 96,9 & 448 & 96,8 & 387 & 51,6 & 89,5 \\
\hline Consentimento cirúrgico & 386 & 94,4 & 414 & 97,4 & 457 & 97,2 & 453 & 97,8 & 428 & 29,3 & 96,7 \\
\hline Avaliação pré-anestésica & 396 & 96,8 & 415 & 97,6 & 461 & 98 & 451 & 97,4 & 431 & 26,4 & 97,5 \\
\hline Sítio cirúrgico demarcado & 389 & 95,1 & 411 & 96,7 & 462 & 98,3 & 450 & 97,2 & 428 & 29,4 & 96,8 \\
\hline $\begin{array}{l}\text { Verificação do equipamento de } \\
\text { anestesiologia e medicação }\end{array}$ & 395 & 96,6 & 422 & 99,3 & 455 & 96,8 & 447 & 96,5 & 430 & 23,5 & 97,3 \\
\hline $\begin{array}{l}\text { Risco de complicações das vias } \\
\text { aéreas }\end{array}$ & 392 & 95,8 & 422 & 99,3 & 463 & 98,5 & 450 & 97,2 & 432 & 27,3 & 97,7 \\
\hline Risco de aspiração & 389 & 95,1 & 418 & 98,4 & 459 & 97,7 & 452 & 97,6 & 430 & 28,1 & 97,2 \\
\hline O paciente possui alergia. & 379 & 92,7 & 410 & 96,5 & 455 & 96,8 & 448 & 96,8 & 423 & 30,6 & 95,7 \\
\hline Faz uso de anticoagulante & 391 & 95,6 & 415 & 97,6 & 456 & 97 & 454 & 98,1 & 429 & 27,4 & 97,1 \\
\hline $\begin{array}{l}\text { Risco de perda sanguínea: adulto > } \\
500 \mathrm{ml} \text {, criança } 7 \mathrm{ml} / \mathrm{kg}\end{array}$ & 392 & 95,8 & 421 & 99,1 & 459 & 97,7 & 455 & 98,3 & 432 & 27,3 & 97,7 \\
\hline Reserva de hemocomponentes & 390 & 95,4 & 417 & 98,1 & 461 & 98,1 & 450 & 97,2 & 430 & 28 & 97,2 \\
\hline Exames de imagem disponíveis & 394 & 96,3 & 413 & 97,2 & 465 & 98,9 & 450 & 97,2 & 431 & 28,3 & 97,4 \\
\hline $\begin{array}{l}\text { Material cirúrgico necessário ao } \\
\text { procedimento em sala }\end{array}$ & 394 & 96,3 & 409 & 96,2 & 459 & 97,7 & 455 & 98,3 & 429 & 28,3 & 97,1 \\
\hline $\begin{array}{l}\text { Revisão da equipe de enfermagem: } \\
\text { esterilização } \\
\text { indicadores), } \\
\text { revisados e montados }\end{array}$ & 382 & 93,4 & 398 & 93,6 & 439 & 93,4 & 449 & 97 & 417 & 27,8 & 94,4 \\
\hline Placa de eletrocautério & 374 & 91,4 & 409 & 96,2 & 449 & 95,5 & 442 & 95,5 & 419 & 29,8 & 94,7 \\
\hline $\begin{array}{l}\text { Profilaxia antimicrobiana foi } \\
\text { realizada nos últimos } 60 \mathrm{~min} .\end{array}$ & 372 & 91 & 398 & 93,6 & 434 & 92,3 & 434 & 93,7 & 410 & 26,2 & 92,7 \\
\hline
\end{tabular}

AQ: Adesão quadrienal; Set: setembro; Out: outubro; Nov: novembro; Dez: dezembro.

Fonte: Autores.

A adesão ao preenchimento do item "procedimento e sítio cirúrgico confirmados com paciente e equipe” na porta de entrada do centro cirúrgico foi de 93,4\%. Na sala de cirurgia, obteve-se maior taxa de adesão $(97,7 \%)$ nos itens "risco de complicações das vias aéreas" e "risco 
Avaliação da adesão ao checklist de cirurgia segura em um hospital universitário $\mid 8$

de perda sanguínea”, os quais apresentaram médias de preenchimento de $432(\mathrm{DP}=27,3)$ questionários. Em contrapartida, uma taxa menor de adesão $(89,5 \%)$ ocorreu no item “equipe em sala sem adornos”, com uma média menor, de 387 (DP=51,6).

\section{Discussão}

Os resultados evidenciaram que a taxa de adesão ao checklist nas unidades de internação variou de 23,3 a $74,4 \%$ e no centro cirúrgico, de 55,2 a 61,2\%. A adesão ao instrumento de cirurgia segura ainda é um desafio, são diversas publicações que reportam o preenchimento incompleto e ineficaz do mesmo., ${ }^{2,10-13}$

Cada item do checklist funciona como um alerta e expressa a possibilidade de prevenção da ocorrência de erros que venham a prejudicar os pacientes. De acordo com a literatura científica, estudo realizado na Austrália verificou, após a implementação da lista de verificação, uma redução das taxas de mortalidade pós-operatória de 1,2 para $0,92 \%$ e do tempo de internação dos pacientes de 5,2 para 4,7 dias. ${ }^{14}$ Em concordância, neste estudo, observou-se uma checagem maior e mais precisa nos itens que, se não conferidos, poderiam causar maiores danos aos pacientes.

Nas unidades de internação, o item “paciente em jejum” apresentou maior taxa de adesão (80,8\%) devido à sua necessidade de checagem, no pré-operatório, para evitar intercorrências de aspiração do suco gástrico durante o procedimento, ${ }^{1}$ bem como o cancelamento da cirurgia. Em outros hospitais, pode-se identificar a adesão favorável $(96,1 \%)$ a esta prática, ratificando o quanto é necessário a checagem para promoção da segurança do paciente. ${ }^{15}$

Em relação ao item “alérgico a medicações”, verificou-se uma adesão razoável de preenchimento $(78,8 \%)$. Diferente do observado em um país africano, em que não houve o preenchimento desse item. ${ }^{16} \mathrm{O}$ paciente, ao manifestar reações alérgicas durante a cirurgia, aumenta as chances de ocorrerem complicações graves, como hipóxia no momento cirúrgico, 
9 | Magnago TSBS, Lanes TC, Gonçalves NGC, Ongaro JD, Almeida FO, Dalmolin GL

além de afetar sua recuperação no pós-operatório. Nesse sentido, é importante que a equipe em sala esteja consciente e confirme com o anestesiologista todas e quaisquer informações acerca das reações alérgicas que o paciente possa apresentar. ${ }^{1}$

Nas unidades de internação, a taxa de adesão do preenchimento do item "Está com pulseira de identificação" foi de 78,3\%. Esse percentual foi inferior ao encontrado em um estudo documental brasileiro, que evidenciou taxas entre 89,1 e $98 \%$ em relação à conferência correta da identificação. ${ }^{15}$ Ao se preencher o nome do paciente de forma incorreta na pulseira de identificação e/ou esquecer de colocá-la no paciente, isso pode induzir à troca de pacientes e/ou erros de procedimentos. É necessário que os profissionais compreendam a importância da identificação correta e quão grave são os problemas que podem afetar os pacientes sem esta prática segura. $^{1}$

A retirada de próteses dentária apresentou menor taxa de adesão nas unidades de internação, seguida pelos itens "uso apenas da camisola" e "adornos retirados", favorecendo o aumento das chances de incidentes com os pacientes, durante a cirurgia. ${ }^{17} \mathrm{~A}$ baixa adesão ao preenchimento desses itens pode estar relacionada à sobrecarga de trabalho proveniente da alta demanda de atividades aliada ao déficit de profissionais, ${ }^{18}$ que por sua vez, pode comprometer a segurança do paciente.

A retirada da prótese evita lesões na cavidade oral durante a intubação, bem como previne náusea e vômitos. Além disso, a verificação do uso da roupa adequada determinada pela instituição e, em especial a retirada de adornos devem ser checados, pois durante o ato cirúrgico pode ocasionar queimaduras na pele devido ao uso do eletrocautério. ${ }^{1}$

$\mathrm{Na}$ porta de entrada do centro cirúrgico, é realizado a confirmação do procedimento e demarcação do local com o paciente. Essa checagem evita trocas de pacientes, cirurgias em local ou procedimento incorretos. ${ }^{1}$ Neste estudo, observou-se elevada $(93,4 \%)$ taxa de adesão ao seu 
Avaliação da adesão ao checklist de cirurgia segura em um hospital universitário $\mid 10$

preenchimento, o que fortalece a segurança do paciente e a realização segura e correta do procedimento. $^{15}$

Quanto à adesão ao preenchimento do checklist na sala de cirurgia, a revisão do risco de perda sanguínea ocorreu em $97,7 \%$ dos pacientes. A adesão pode estar relacionada ao risco de hemorragias e de choque hipovolêmico, sempre presentes no ato cirúrgico. A partir dessa avaliação, a equipe do banco de sangue consegue providenciar e armazenar bolsas de sangue, com antecedência. Estudo brasileiro evidenciou essa checagem em 99,1\% dos procedimentos cirúrgicos. ${ }^{11}$ Em concordância sobre a importância da avaliação, identificou-se no estudo realizado na República do Congo a perda sanguínea acentuada em $86 \%$ das cirurgias. ${ }^{19}$

Outros fatores importantes preenchidos pelos profissionais no centro cirúrgico foram seguintes: risco de aspiração e complicações das vias aéreas $(97,7 \%)$ e avaliação pré-anestésica (97,4\%). Neste estudo, os itens diretamente relacionados com a equipe anestésica possuem uma taxa de adesão maior, talvez pelo fato de esta equipe já possuir historicamente como rotina a conferência sistemática de itens essenciais ao procedimento anestésico. Pacientes pediátricos de hospital londrino, em $89 \%$ dos casos, apresentaram um plano de verificação das vias aéreas, o que sinalizou a preocupação dos profissionais em relação a possíveis complicações relacionadas a esse item..$^{20}$

A função respiratória no período da indução anestésica é um fator complexo de se manter. Nesse momento, os componentes anestésicos atuam diretamente na diminuição do tônus muscular das vias aéreas. Isso auxilia a atenuar os reflexos e a impossibilitar a realização da respiração própria pelo paciente, o que pode causar hipóxia durante a estimulação anestésica. ${ }^{1}$

Para reduzir as complicações provenientes da cirurgia que envolve, principalmente, o trato respiratório e perda sanguínea é necessário que a equipe revise o manejo dos pacientes e sua transferência para a sala de recuperação. Além de inserir intervenções que sensibilizem os 
11 | Magnago TSBS, Lanes TC, Gonçalves NGC, Ongaro JD, Almeida FO, Dalmolin GL

profissionais a realizar o manejo e melhorar a adesão ao preenchimento; como sucedeu em um hospital do Reino Unido, que obteve um progresso de 58 a 72\% de adesão às preocupações com a recuperação do paciente..$^{20}$

O checklist institucional contempla a conferência dos instrumentais e verificação do material cirúrgico. Ao contrário das demais situações, evidenciaram-se baixos índices em relação à contagem de instrumentais, semelhante a um estudo do Distrito Federal brasileiro, que apresentou apenas $2 \%$ de adesão. ${ }^{21} \mathrm{~A}$ contagem de materiais é de suma relevância, tendo em vista a complexidade do dano que pode ser causado pelo esquecimento de material cirúrgico no interior do paciente. ${ }^{15}$

\section{Conclusão}

A taxa de adesão ao checklist de cirurgia segura foi baixa, variando de 23,3 a $74,4 \%$ nas unidades de internação e de 55,2 a 61,2\% no centro cirúrgico. Nas unidades de internação, o item “paciente em jejum” obteve a maior média de adesão (357; DP=20,9), com taxa de 80,8\%. Na porta de entrada do centro cirúrgico o item "procedimento e sítio cirúrgico confirmados com paciente e equipe" apresentou média de $413(\mathrm{DP}=32,2)$ com taxa de 93,4\% de adesão ao seu preenchimento. Na sala de cirurgia, os itens "risco de complicações das vias aéreas e "risco de perda sanguínea” apresentaram média de preenchimento do checklist de $432(\mathrm{DP}=27,3)$ e taxa de adesão 97,7\%. Diante da baixa taxa de adesão ao checklist, notou-se a importância de novos estudos que desenvolvam e implementem estratégias que possam auxiliar no seu preenchimento, com intuito de promover e melhorar a qualidade e segurança do paciente em procedimentos cirúrgicos.

\section{Referências}


1. Organização Mundial da Saúde (OMS). Segundo desafio global para a segurança do paciente: cirurgias seguras salvam vidas (orientações para cirurgia segura da OMS) [Internet]. 2009 [acesso em 2018 dez 20]. Disponível em: http://bvsms.saude.gov.br/bvs/publicacoes/seguranca_paciente_cirurgias_seguras_salvam_vidas.pdf

2. Gillespie BM, Harbeck E, Lavin J, Gardiner T, Withers TK, Marshall AP. Using normalisation process theory to evaluate the implementation of a complex intervention to embed the surgical safety checklist. BMC Health Serv Res [Internet]. 2018 [acesso em 2019 set 10];18(170). Disponível em: https://www.ncbi.nlm.nih.gov/pmc/articles/PMC5845378/ doi: 10.1186/s12913-018-2973-5

3. Ferraz EM. A cirurgia segura: uma exigência do século XXI. Rev Col Bras Cir [Internet]. 2009 [acesso em 2018 maio 20];36(4):281-2. Disponível em: http://www.scielo.br/pdf/rcbc/v36n4/a01v36n4.pdf

4. Munhoz OL, Andolhe R, Magnago TSBS, Dalmolin GL, Pasa TS. Profile of patients and incidents in a surgical clinic unit. Rev Enferm UFPE On Line [Internet]. 2018 [acesso em 2019 set 10];12(2):416-23. Disponível em: https://periodicos.ufpe.br/revistas/revistaenfermagem/article/view/230813 doi:10.5205/1981-8963-v12i2a230813p416-423-2018

5. Skarsgard ED. Recommendations for surgical safety checklist use in Canadian children's hospitals. Can J Surg [Internet]. 2016 [acesso em 2018 jan 02];59(3):161-6. Disponível em: https://www.ncbi.nlm.nih.gov/pmc/articles/PMC4982860/ doi: 10.1503/cjs.016715

6. Alpendre FT, Cruz EDA, Dyniewicz AM, Mantovani MF, Silva AEBC, Santos GS. Cirurgia segura: validação de checklist pré e pós-operatório. Rev Latinoam Enferm [Internet]. 2017 [acesso em 2018 out 08];25:e2907. Disponível em: http://www.scielo.br/pdf/rlae/v25/pt_0104-1169-rlae-25-e2907.pdf doi: $10.1590 / 1518-8345.1854 .2907$

7. Criado JVN, Dias BF, Carmo TG. Processo de implementação do protocolo de cirurgia segura. Rev Cuba Enferm [Internet]. 2017 [acesso em 2018 jul 21];33(1). Disponível em: http://www.revenfermeria.sld.cu/index.php/enf/article/view/1028/240

8. Bolfarine H, Bussab WO. Elementos de amostragem. São Paulo (SP): Blucher; 2005.

9. Brasil. Ministério da Saúde. Conselho Nacional de Saúde. Resolução no 466, de 12 de dezembro de 2012. Diretrizes e normas regulamentadoras de pesquisa envolvendo seres humanos. Brasília (DF): Ministério da Saúde; 2012.

10. Brindle ME, Henrich N, Foster A, Marks S, Rose M, Welsh R, et al. Implementation of surgical debriefing programs in large health systems: an exploratory qualitative analysis. BMC Health Serv Res [Internet]. 2018 [acesso em 2019 set 10];18(210). Disponível em: https://www.ncbi.nlm.nih.gov/pmc/articles/PMC5870386/ doi:10.1186/s12913-018-3003-3

11. Ribeiro HCTC, Quites HFO, Bredes AC, Sousa KAS, Alves M. Adesão ao preenchimento do checklist de segurança cirúrgica. Cad Saúde Pública [Internet]. 2017 [acesso em 2019 set 10];33(10):e00046216. 
13 | Magnago TSBS, Lanes TC, Gonçalves NGC, Ongaro JD, Almeida FO, Dalmolin GL

Disponível em: http://www.scielo.br/scielo.php?script=sci_arttext\&pid=S0102311X2017001005011\&lng=pt\&tlng=pt doi: 10.1590/0102-311x00046216

12. Maziero ECS, Silva AEBC, Mantovani MF, Cruz EDA. Adesão ao uso de um checklist cirúrgico para segurança do paciente. Rev Gaúch Enferm [Internet]. 2015 [acesso em 2018 jan 03];36(4):14-20. Disponível em: http://www.scielo.br/pdf/rgenf/v36n4/pt_1983-1447-rgenf-36-04-00014.pdf doi:10.1590/19831447.2015.04.53716

13. Almeida RE, Rodrigues MCS. Execução da lista de verificação de segurança cirúrgica em operações pediátricas: avaliação da conformidade. Rev Gaúch Enferm [Internet]. 2019 [acesso em 2019 set 10];40(N Esp):e20180270. Disponível em: http://www.scielo.br/scielo.php?pid=S198314472019000200414\&script=sci_abstract\&tlng=es doi:10.1590/1983-1447.2019.20180270

14. Jager E, Gunnarsson R, Ho YH. Implementation of the world health organization surgical safety checklist correlates with reduced surgical mortality and length of hospital admission in a high-income country. World J Surg [Internet]. 2019 [acesso em 2019 set 10];43:117-124. Disponível em: https://link.springer.com/article/10.1007\%2Fs00268-018-4703-x doi:10.1007/s00268-018-4703-x

15. Marquioni FSN, Moreira TR, Diaz FBBS, Ribeiro L. Cirurgia segura: avaliação da adesão ao checklist em hospital de ensino. Rev SOBECC [Internet]. 2019 [acesso em 2019 set 10];24(1):22-30. Disponível em: https://revista.sobecc.org.br/sobecc/article/view/22/pdf doi: 10.5327/Z1414-4425201900010006

16. Ellis R, Nor AIM, Pimentil I, Bitew Z, Moore J. Improving surgical and anaesthesia practice: review of the use of the WHO safe surgery checklistin Felege Hiwot referral hospital, Ethiopia. BMJ Qual Improv Rep [Internet]. 2017 [acesso em 2018 dez 15];6(1):u207104.w6251. Disponível em: https://www.ncbi.nlm.nih.gov/pmc/articles/PMC5337669/pdf/bmjqir.u207104.w6251.pdf doi: 10.1136/bmjquality.u207104.w6251

17. Bisinotto FMB, Dezena RA, Martins LB, Galvão MC, Martins Sobrinho J, Calçado MS. Burns related to electrosurgery: report of two cases. Braz J Anesthesiol [Internet]. 2017 [acesso em $2018 \mathrm{dez}$ 18];67(5):527-34. Disponível em: https://www.sciencedirect.com/science/article/pii/S0034709416300241?via\%3Dihub doi: https://doi.org/10.1016/j.bjan.2016.03.003

18. Padilha KG, Barbosa RL, Andolhe R, Oliveira EM, Ducci AJ, Bregalda RS, et al . Carga de trabalho de enfermagem, estresse/burnout, satisfação e incidentes em Unidade de Terapia Intensiva de trauma. Texto \& Contexto Enferm [Internet]. 2017 [acesso em 2019 set 10];26(3):e1720016. Disponível em: http://www.scielo.br/scielo.php?script=sci_arttext\&pid=S0104-07072017000300322\&lng=pt doi: 10.1590/0104-07072017001720016

19. White MC, Peterschmidt J, Callahan J, Fitzgerald EJ, Close KL. Interval follow up of a 4-day pilot program to implement the WHO surgical safety checklistat a congolese hospital. Global Health 
Avaliação da adesão ao checklist de cirurgia segura em um hospital universitário | 14

[Internet]. 2017 [acesso em 2018 nov 29];13:42. Disponível em: https://www.ncbi.nlm.nih.gov/pmc/articles/PMC5492505/ doi:10.1186/s12992-017-0266-0

20. Keijzer WW, Agha RA, Greig A. WHO safer surgery checklist compliance amongst paediatric emergency plastic surgery patients in an UK hospital. Ann Med Surg (Lond) [Internet]. 2017 [acesso em 2018 nov 18];21:49-52. Disponível em:https://www.ncbi.nlm.nih.gov/pmc/articles/PMC5537375/ doi: 10.1016/j.amsu.2017.07.049

21. Santana HT, Freitas MR, Ferraz EM, Evangelista MSN. WHO safety surgical checklistimplementation evaluation in public hospitals in the brazilian Federal District. J Infect Public Health [Internet]. 2016 [acesso em 2018 out 08];9(5):586-99. Disponível em: https:/www.sciencedirect.com/science/article/pii/S1876034116000101?via\%3Dihub doi: 10.1016/j.jiph.2015.12.019

\section{Autor correspondente}

Tânia Solange Bosi de Souza Magnago

E-mail: tmagnago@terra.com.br

Endereço: Av. Roraima, 1000 - Camobi, Santa Maria - RS

CEP: $97105-900$

\section{Contribuições de Autoria}

\section{1 - Tânia Solange Bosi de Souza Magnago}

Contribuições: Concepção e planejamento do projeto; análise e interpretação dos dados; Redação do artigo e revisão crítica relevante do conteúdo intelectual; Aprovação final da versão a ser publicada.

\section{2 - Taís Carpes Lanes}

Contribuições: Análise e interpretação dos dados; Redação do artigo e revisão crítica relevante do conteúdo intelectual; Aprovação final da versão a ser publicada.

\section{3 - Naiane Glaciele da Costa Gonçalves}

Contribuições: Redação do artigo e revisão crítica relevante do conteúdo intelectual; Aprovação final da versão a ser publicada.

\section{4 - Juliana Dal Ongaro}

Contribuições: Redação do artigo e revisão crítica relevante do conteúdo intelectual; Aprovação final da versão a ser publicada.

\section{4 - Franciele Ormizinda Almeida}

Contribuições: Redação do artigo e revisão crítica relevante do conteúdo intelectual; Aprovação final da versão a ser publicada. 
15 | Magnago TSBS, Lanes TC, Gonçalves NGC, Ongaro JD, Almeida FO, Dalmolin GL

6 - Graziele de Lima Dalmolin

Contribuições: Redação do artigo e revisão crítica relevante do conteúdo intelectual; Aprovação final da versão a ser publicada.

\section{Como citar este artigo}

Magnago TSBS, Lanes TC, Gonçalves NGC, Ongaro JD, Almeida FO, Dalmolin GL. Avaliação da adesão ao checklist de cirurgia segura em um hospital universitário. Rev. Enferm. UFSM. 2019 [Acesso em: Anos Mês Dia];vol9 e63: 1-15. DOI:https://doi.org/10.5902/2179769236780 